\title{
Competition of Superconductivity and Antiferromagnetism in a d-Wave Vortex Lattice
}

\author{
Amit Ghosal, Catherine Kallin and A. John Berlinsky \\ Department of Physics and Astronomy, McMaster University, Hamilton, Ontario, L8S 4M1, Canada
}

The d-wave vortex lattice state is studied within the framework of Bogoliubov-de Gennes (BdG) mean field theory. We allow antiferromagnetic (AFM) order to develop selfconsistently along with d-wave singlet superconducting (dSC) order in response to an external magnetic field that generates vortices. The resulting AFM order has strong peaks at the vortex centers, and changes sign, creating domain walls along lines where $\nabla \times j_{s} \approx 0$. The length scale for decay of this AFM order is found to be much larger than the bare d-wave coherence length, $\xi$. Coexistence of dSC and AFM order in this system is shown to induce $\pi$-triplet superconducting order. Competition between different orders is found to suppress the local density of states at the vortex center and comparison to recent experimental findings is discussed.

\section{INTRODUCTION}

Many of the anomalous properties of high $T_{c}$ superconductors (SC) are believed to be due to the competition between different kinds of orders. The electronic structure of vortices in the cuprates is one striking example of behavior which is not understood and which may be influenced by competing or coexisting order parameters. The electronic structure of s-wave vortices has been studied extensively by both theoretical [1,2] and experimental [3] methods which established the existence of localized vortex states with very low energy. The situation is more complex for the case of d-wave SC due to the presence of nodes in the energy gap. Earlier theoretical results of Wang and MacDonald [4], performed within the framework of Bogliubov-de Gennes (BdG) calculations, predicted a strong and broad zero bias conductance peak (ZBCP) in the local density of states (LDOS) at the vortex core. This ZBCP was shown [5] to display a four-fold symmetric "star-shape", oriented towards the gap nodes and decaying as a power law with distance from the vortex core. In contrast, experiments present a completely different picture. Tunneling studies of both YBCO [6] and BSCCO [7] show no evidence of a ZBCP. Furthermore, structure is observed in the low energy differential conductance data which has been interpreted as the existence of low energy quasiparticle states at $\pm 5.5 \mathrm{meV}$ (for $\mathrm{YBCO}$ ) and at $\pm 7 \mathrm{meV}$ (for BSCCO). These states persist to long distances - a size much larger than that of the vortex core.

There have been a number of different proposals for reconciling this contradiction between theoretical predictions and experimental findings, including effects of strong correlations and competing order parameters. The role of strong correlations was emphasized by Franz and Tesanovic [8] who argued that the correct description of the normal state of high $T_{c}$ cuprates is a doped Mott insulator and not a simple metal (as in BCS theory). Hence, at the vortex core, where superconductivity is suppressed, a gapped insulator should be expected. In this case, the pseudogap behavior of the tunneling results at the core center is a result of spin-charge separation. To address the correlation effects on the structure of an isolated vortex, Han and Lee [10] studied the $t-J$ model using resonant valence bond mean field theory. Their main result is the prediction of two types of vortex core - insulating or metallic, depending on the model parameters. They also found evidence of staggered current patterns [9] in the vortex core. The pairing order parameter near the vortex core was found to have $d_{x^{2}-y^{2}}+i d_{x y}$ symmetry [10,11. A different argument for the absence of the ZBCP invokes the structure of the c-axis tunneling matrix element [12]. In a recent study, Berthod and Giovannini 13 showed that with increasing correlation strength the BCS core states become suppressed and the spectra inside and outside the vortex core become similar.

An alternative scenario has been presented in which antiferromagnetic (AFM) order is generated near the vortex core. Arovas and collaborators 14 first studied this problem within the framework of $\mathrm{SO}(5)$ theory and predicted that the vortex core becomes antiferromagnetic, and hence insulating, at low temperature and relatively low doping. Subsequently, a BdG calculation of $\mathrm{SO}(5)$ vortices 15 found that the AFM order, although itself gapless within this model, suppressed the ZBCP. Recent works of Sachdev and collaborators [16 argue that the system undergoes a quantum phase transition from a pure d-wave superconducting (dSC) phase to a mixture of dSC and spin density wave (SDW) phase as a function of the external magnetic field. In this theory the dynamic spin fluctuations in dSC are pinned by the vortex cores. More recently Zhu and Ting [17 have studied a d-wave vortex lattice, allowing for AFM order, using BdG mean field theory. They find AFM order which alternates in sign from one vortex to the next, and a splitting of the ZBCP due to the AFM order.

There have been a large number of recent experiments which suggest the emergence of AFM or SDW order on top of the dSC order in the cuprates in an external magnetic field. Using inelastic neutron scattering, Lake et al. 
[18 found that the magnetic field induces a large increase in the spectral density of low energy spin fluctuations in LSCO near optimal doping. This demonstrates the existence of a fluctuating magnetisation density with a periodicity of 8 lattice spacings along the direction of the $\mathrm{Cu}-\mathrm{O}$ bonds. Scanning tunneling microscopy (STM) on slightly overdoped BSCCO [19] in the mixed state shows a charge modulation in the LDOS data that has a periodicity of 4 lattice spacings - a result consistent with the neutron scattering study since the periodicity of charge modulation is one half of that of the SDW modulation. Field induced enhancement of the Bragg peak intensity has also been observed by Khaykovich et al. [20], in an elastic neutron scattering study of $\mathrm{La}_{2} \mathrm{CuO}_{4+\mathrm{y}}$, indicating a field induced static AFM order. High-field nuclear magnetic resonance (NMR) studies of YBCO 21 have found evidence for strong antiferromagnetic fluctuations inside the core. Muon spin rotation measurements [22] have also been interpreted as evidence of static magnetism in the vortex core of Ortho-II $\mathrm{YBa}_{2} \mathrm{Cu}_{3} \mathrm{O}_{6.5}$. Understanding the charge and spin modulation in the vortex lattice state is currently under intensive theoretical investigation 23 25].

Motivated by the discrepancy between theoretical predictions and experimental observations, we study the structure and spectrum of a d-wave vortex lattice using a BdG mean field theory which allows both static AFM order and dSC order in a self-consistent manner. Our main results are: (1) In the lowest energy configuration, AFM order has the same periodicity as the vortex lattice, in contrast to earlier studies [17], although there are antiphase domain walls in the AFM order within the unit cell; (2) triplet SC order is induced in the presence of coexisting AFM and dSC, which approximately satisfies the constraint predicted by Zhang 26]; (3) due to the presence of AFM order, the LDOS exhibits structure which persists well beyond the vortex core size.

The organization of the paper is as follows. In Sec. II, we describe the class of models we study, and we also elaborate on the BdG method. Sec. III discusses our results on the spatial structures of different order parameters in the mixed state, and Sec. IV discusses the LDOS results. Finally, we present conclusions in Sec. IV.

\section{MODEL AND METHOD}

We describe a $2 \mathrm{D}$ d-wave $\mathrm{SC}$ in an external magnetic field by the following Hamiltonian :

$$
\mathcal{H}=\mathcal{K}+\mathcal{H}_{\text {int }}+\mathcal{H}_{\mu},
$$

where the kinetic energy,

$$
\mathcal{K}=-t \sum_{<i j>, \alpha}\left(e^{i \phi_{i j}} c_{i \alpha}^{\dagger} c_{j \alpha}+\text { h.c. }\right),
$$

describes electrons, with spin $\alpha$ at site $i$ created by $c_{i \alpha}^{\dagger}$, hopping between nearest-neighbors $\langle i j\rangle$ on a square lat- tice. The Peierl's phase factor $\phi_{i j}=\left(\pi / \Phi_{0}\right) \int_{\mathbf{r}_{i}}^{\mathbf{r}_{j}} \mathbf{A}(\mathbf{r}) \cdot d \mathbf{r}$, with the superconducting flux quantum $\Phi_{0}=h c / 2 e$, denotes the presence of an external magnetic field produced by the vector potential $\mathbf{A}(\mathbf{r})$. The interaction term in Eq. (1),

$$
\mathcal{H}_{\text {int }}=J \sum_{<i j>}\left(\mathbf{S}_{i} \cdot \mathbf{S}_{j}-\frac{1}{4} n_{i} n_{j}\right),
$$

is chosen to generate both antiferromagnetic and d-wave superconducting orders at the mean field level. Here the spin operator is $S_{i}^{a}=c_{i \alpha}^{\dagger} \sigma_{\alpha \beta}^{a} c_{i \beta}$, where the $\sigma^{a}$ are Pauli matrices, and the density operator is $n_{i \alpha}=c_{i \alpha}^{\dagger} c_{i \alpha}$ with $n_{i}=n_{i \uparrow}+n_{i \downarrow}$. An onsite repulsion term of the form $U \sum_{i} n_{i \uparrow} n_{i \downarrow}$ can be added to $\mathcal{H}_{\text {int }}$ to ensure the absence of any onsite s-wave pairing, however, we found that it is not necessary because such induced s-wave pairing is vanishingly small for our parameter regime. The chemical potential part of the Hamiltonian, $\mathcal{H}_{\mu}=-\mu \sum_{i} n_{i}$, is used to fix the total density of the system to the desired value.

It is commonly believed that this simple model, in the limit of no double occupancy of any site, is adequate to describe the strongly correlated cuprates at low temperatures. A mean field treatment of this model, while missing the strong correlation effects, may still usefully describe the interplay between static antiferromagnetic order and d-wave superconductivity 27.

A mean field decomposition of the above model leads to the following effective Hamiltonian

$$
\begin{aligned}
\mathcal{H}_{\mathrm{eff}} & =-\sum_{i, \delta, \alpha}\left(t+\tau_{i \sigma}^{\delta}\right) e^{i \phi_{i j}} c_{i \alpha}^{\dagger} c_{i+\delta \alpha} \\
& -\sum_{i, \alpha}\left(\mu_{i}-\eta_{i}+\alpha e^{i \mathbf{Q} \cdot \mathbf{r}_{i}} \sum_{\delta} m_{i+\delta}\right) n_{i \alpha} \\
& +\sum_{i, \delta}\left(\Delta_{i}^{\delta}\left[c_{i+\delta \uparrow}^{\dagger} c_{i \downarrow}^{\dagger}+c_{i \uparrow}^{\dagger} c_{i+\delta \downarrow}^{\dagger}\right]+\text { h.c. }\right)
\end{aligned}
$$

where $\tau_{i \sigma}^{\delta}$ is the Fock shift that renormalizes the hopping amplitude $t$ and $\eta_{i}$ is the Hartree shift that renormalizes the chemical potential $\mu$. Here $\delta= \pm \hat{x}, \pm \hat{y}$, the four nearest neighbours of any site, and $\phi_{i}^{\delta}$ is same as $\phi_{i j}$ with $\mathbf{j}=\mathbf{i}+\delta$. In the above equation the local dSC $\left(\Delta_{i}^{\delta}\right)$ and AFM $\left(m_{i}\right)$ order parameters, and the Hartree $\left(\eta_{i}\right)$ and Fock $\left(\tau_{i \sigma}\right)$ shifts satisfy the following self-consistency conditions:

$$
\begin{aligned}
\Delta_{i}^{\delta} & =\frac{J}{4}\left(\left\langle c_{i+\delta \downarrow} c_{i \uparrow}\right\rangle+\left\langle c_{i \downarrow} c_{i+\delta \uparrow}\right\rangle\right) \\
m_{i} & =\frac{J}{2}\left(\left\langle n_{i \uparrow}\right\rangle-\left\langle n_{i \downarrow}\right\rangle\right) e^{i \mathbf{Q} \cdot \mathbf{r}_{i}} \\
\tau_{i \sigma}^{\delta} & =\frac{J}{2}\left\langle c_{i+\delta,-\sigma}^{\dagger} c_{i,-\sigma}\right\rangle \\
\eta_{i} & =-\frac{J}{4} \sum_{\delta}\left\langle n_{i+\delta}\right\rangle
\end{aligned}
$$


where $\mathbf{Q}=(\pi, \pi)$ is the antiferromagnetic wave vector. The Hamiltonian in Eq. (何) has been studied for a range of model parameters. In the following we will report results primarily from our calculations at zero temperature $(T=0)$ with parameters $J / t=1.15$ and $\langle n\rangle=0.875$. In the absence of an external magnetic field, these parameters result in a uniform d-wave gap, $\Delta_{\max } \sim 0.36 t$ (which is $8 \Delta^{\delta}$ in our notation), with superconducting coherence length $\xi_{0} \approx 4 a$ (where $a$ is the lattice spacing), a value consistent with high $T_{c}$ cuprates.

We have also studied an extended Hubbard model where the dSC is generated by a nearest neighbour attractive interaction of the form: $\mathcal{H}_{\text {int }}=$ $-J^{\prime} \sum_{<i j>, \alpha, \alpha^{\prime}} n_{i \alpha} n_{j \alpha^{\prime}}$. Self consistent calculation on such a model will generate a triplet superconducting order in addition to a singlet dSC order when decomposed in a mean field manner. Also, such an extended Hubbard interaction does not by itself generate AFM order, which has to be incorporated into the model using an additional interaction parameter [17]. By contrast, both dSC and AFM orders are generated from the single $J$-term in Eq. (3) in our calculations, thereby putting both orders on an equal footing. Another problem of the extended Hubbard model is that, the strength of the onsite repulsion, $U$, required to generate AFM order has to be finetuned to study the interplay of the two competing orders. Non-zero AFM order develops only for $U \geq U_{c}$, a result consistent with earlier findings [17]. On the other hand a strong $U$ would generate strong antiferromagnetism that would in turn kill d-wave superconductivity. Except for these limitations, if the triplet order is ignored, the mean field decomposition of the extended Hubbard model generates an effective Hamiltonian similar to that given by Eq. (雨), but with different values for the Hartree and Fock shifts. We have checked that the qualitative physics described by the extended Hubbard model at the mean field level remains similar to that for Eq. (4), reported in this work.

$\mathcal{H}_{\text {eff }}$ can be diagonalized using Bogoliubov tranformations,

$$
c_{i \alpha}=\sum_{n}\left\{u_{n, \alpha}(i) \gamma_{n, \alpha}-\alpha v_{n, \alpha}^{*}(i) \gamma_{n,-\alpha}^{\dagger}\right\}
$$

leading to the usual BdG equations

$$
\left(\begin{array}{cc}
\hat{\xi}-\alpha \hat{M} & \hat{\Delta} \\
\hat{\Delta}^{*} & -\hat{\xi}^{*}-\alpha \hat{M}
\end{array}\right)\left(\begin{array}{c}
u_{n, \alpha} \\
v_{n,-\alpha}
\end{array}\right)=E_{n}\left(\begin{array}{c}
u_{n, \alpha} \\
v_{n,-\alpha}
\end{array}\right)
$$

where $\hat{\xi} u_{n, \alpha}(i)=-\sum_{\delta} \tilde{t_{i}^{\delta}} u_{n, \alpha}(i+\delta)-\tilde{\mu_{i}} u_{n, \alpha}(i)$, $\hat{M} u_{n, \alpha}(i)=\left(\sum_{\delta} m_{i+\delta}\right) e^{i \mathbf{Q} \cdot \mathbf{r}_{\mathbf{i}}} u_{n, \alpha}(i)$ and $\hat{\Delta} u_{n, \alpha}(i)=$ $\sum_{\delta}\left(\Delta_{i}^{\delta}+\Delta_{i+\delta}^{-\delta}\right) u_{n, \alpha}(i+\delta)$. One can numerically diagonalize Eq. (7) directly. However, exploiting the magnetic translation symmetry of the effective Hamiltonian allows one to treat much larger system sizes. This procedure was discussed by Wang and MacDonald [⿰]. . However, since our model and resulting BdG equations differ somewhat, we describe below the block diagonalization procedure we use.
We assume that our system is made up of a periodic array of identical magnetic unit cells of size $N_{x} \times N_{y}$. For our case we take $N_{x}=N_{y} / 2$, a rectangular magnetic unit cell enclosing two superconducting flux quanta, and work in the Landau gauge, $\mathbf{A}(\mathbf{r})=(0, H x)$. In this gauge the magnetic translation operators are $\left\langle\mathbf{r}\left|\mathcal{T}_{R}\right| \mathbf{r}^{\prime}\right\rangle=$ $\delta_{\mathbf{r}, \mathbf{r}+\mathbf{R}} e^{-i b R_{x}\left(i_{y}+R_{y}\right)}$, where the parameter $b$ is defined as $b \equiv H / \Phi_{0}=2 \pi / N_{x} N_{y}$. Since $\left[\mathcal{H}_{\text {eff }}, \mathcal{T}_{R}\right]=0$, the eigenstates of $\mathcal{T}_{R}$ can be used to block diagonalize $\mathcal{H}_{\text {eff }}$. The transformations that block diagonalize the BdG equations in the magnetic wave vector, $\mathbf{k}$, are

$$
\begin{aligned}
& u_{i, \alpha}^{n}(\mathbf{R})=e^{i \mathbf{k} \cdot \mathbf{R}} e^{-i b\left(i_{y}+R_{y}\right) R_{x}} u_{i, \alpha}^{n}(\mathbf{k}) \\
& v_{i, \alpha}^{n}(\mathbf{R})=e^{i \mathbf{k} \cdot \mathbf{R}} e^{i b\left(i_{y}+R_{y}\right) R_{x}} v_{i, \alpha}^{n}(\mathbf{k})
\end{aligned}
$$

where $k_{x}=\frac{2 \pi m_{x}}{p N_{x}}$, with $m_{x}=0,1,2, \ldots,(p-1)$ and $k_{y}=\frac{2 \pi m_{y}}{q N_{y}}$, with $m_{y}=0,1,2, \ldots,(q-1)$. The eigenvalue problem for a system of size $p N_{x} \times q N_{y}$ reduces to $p \times q$ eigenvalue matrix equations, each for a system of size $N_{x} \times N_{y}$. The block diagonal BdG matrix of Eq. (7) becomes, for each $\mathbf{k}$,

$$
\begin{array}{rc}
\left(\begin{array}{cc}
\hat{\xi}_{\alpha}(\mathbf{k})-\alpha \hat{M}(\mathbf{k}) & \hat{\Delta}(\mathbf{k}) \\
\hat{\Delta}^{*}(\mathbf{k}) & -\hat{\xi}_{\alpha}^{*}(\mathbf{k})-\alpha \hat{M}(\mathbf{k})
\end{array}\right)\left(\begin{array}{c}
u_{i, \alpha}^{n}(\mathbf{k}) \\
v_{i,-\alpha}^{n}(\mathbf{k})
\end{array}\right) \\
=E_{\alpha}^{n}(\mathbf{k})\left(\begin{array}{c}
u_{i, \alpha}^{n}(\mathbf{k}) \\
v_{i,-\alpha}^{n}(\mathbf{k})
\end{array}\right)
\end{array}
$$

Here the operators (in the bulk) are defined as

$$
\begin{aligned}
\hat{\xi}_{\alpha}(\mathbf{k}) u_{i, \alpha}^{n}(\mathbf{k}) & =-\sum_{\delta}\left\{t+\frac{J}{2}\left\langle c_{i+\delta,-\alpha}^{\dagger} c_{i,-\alpha}\right\rangle\right\} e^{i \phi_{i}^{\delta}} u_{i+\delta, \alpha}^{n}(\mathbf{k}) \\
\hat{M}(\mathbf{k}) u_{i, \alpha}^{n}(\mathbf{k}) & =\left\{\sum_{\mathbf{q}, n^{\prime}} m_{\mathbf{q} n^{\prime}}(i)\right\} u_{i, \alpha}^{n}(\mathbf{k}) \\
\hat{\Delta}(\mathbf{k}) v_{i,-\alpha}^{n}(\mathbf{k}) & =2 \sum_{\delta}\left\{\sum_{\mathbf{q}, n^{\prime}} \Delta_{\mathbf{q} n^{\prime}}^{\delta}\right\} v_{i+\delta,-\alpha}^{n}(\mathbf{k})
\end{aligned}
$$

In this 'repeated zone scheme' the self-consistency conditions, Eq. (5), can be rewritten as

$$
\begin{aligned}
\Delta_{\mathbf{q} n}^{\delta}(i) & =\frac{J}{4 \mathcal{N}}\left[\left\{u_{j, \downarrow}^{n}(\mathbf{q}) v_{i, \uparrow}^{n *}(\mathbf{q})+u_{i, \downarrow}^{n}(\mathbf{q}) v_{j, \uparrow}^{n *}(\mathbf{q})\right\}\left(1-f_{\mathbf{q} n \downarrow}\right)\right. \\
& \left.+\left\{u_{i, \uparrow}^{n}(\mathbf{q}) v_{j, \downarrow}^{n *}(\mathbf{q})+u_{j, \uparrow}^{n}(\mathbf{q}) v_{i, \downarrow}^{n *}(\mathbf{q})\right\} f_{\mathbf{q} n \uparrow}\right] \\
m_{\mathbf{q} n}(i) & =\frac{U}{2 \mathcal{N}} e^{i \mathbf{Q} \cdot \mathbf{r}_{i}}\left[\left|u_{i, \uparrow}^{n}(\mathbf{q})\right|^{2} f_{\mathbf{q} n \uparrow}-\left|u_{i, \downarrow}^{n}(\mathbf{q})\right|^{2} f_{\mathbf{q} n \downarrow}\right. \\
& \left.+\left|v_{i, \uparrow}^{n}(\mathbf{q})\right|^{2}\left(1-f_{\mathbf{q} n \downarrow}\right)-\left|v_{i, \downarrow}^{n}(\mathbf{q})\right|^{2}\left(1-f_{\mathbf{q} n \uparrow}\right)\right]
\end{aligned}
$$

where $j=i+\delta$ and $\mathcal{N}=p \times q$, the total number of magnetic unit cells. Note that, for our choice of gauge, the matrices $\hat{\xi}_{\alpha}(\mathbf{k}), \hat{M}(\mathbf{k})$ and $\hat{\Delta}(\mathbf{k})$ are $\mathbf{k}$-independent for the bulk of the system, as can be seen from Eq. (10). However $\mathbf{k}$-dependence enters through the boundary terms (See Appendix).

Starting with an initial guess for all local variables (dSC order, AFM order and Hartree and Fock shifts) and for 
the value of $\mu$, we solve for all eigenvalues and eigenvectors of the BdG matrix for each $\mathbf{k}$, in terms of which all local orders are recalculated using self-consistency conditions Eq. (12). This process is repeated until a convergence is obtained in these variables and also in $\mu$ which fixes the total density to the desired value of 0.875 .

\section{RESULTS}

\section{A. Spatial structure of local order parameters}

In this section we discuss the structure of the dSC, AFM and charge density orders, as well as of the $\pi$-triplet $\mathrm{SC}$ order which is induced in the presence of coexisting AFM and dSC orders.

d-wave Pairing Amplitude: For our lattice BdG calculation we defined a d-wave pairing amplitude $\Delta^{\delta}(\mathbf{i}, \mathbf{R})$ following Eq. (5) that lives on bonds. We can also define a gauge invariant site dependent singlet d-wave order parameter in our model as,

$$
\Delta_{i}=\frac{1}{4}\left[\Delta^{\hat{x}}(\mathbf{r})+\Delta^{-\hat{x}}(\mathbf{r})-e^{-i b x} \Delta^{\hat{y}}(\mathbf{r})-e^{i b x} \Delta^{-\hat{y}}(\mathbf{r})\right]
$$

where $r \equiv(\mathbf{i}, \mathbf{R})$. In the left panel of Fig. (1) we plot the magnitude of $\Delta_{i}$ on half of a magnetic unit cell. The spatial dependence of $\Delta_{i}$ is very similar to its behavior in the absence of AFM order (not shown here). The size of the vortex core, $\xi$, is similar for the two cases, although the initial slope inside the core is somewhat smaller in the case with AFM order. This is what one would expect within Ginzburg-Landau theory [30,31].

The presence of the vortex lattice induces an extended s-wave order $\left(\Delta_{i}^{s}\right)$ around vortex in a self-consistent manner; defined as:

$$
\Delta_{i}^{s}=\left[\Delta^{\hat{x}}(\mathbf{r})+\Delta^{-\hat{x}}(\mathbf{r})+e^{-i b x} \Delta^{\hat{y}}(\mathbf{r})+e^{i b x} \Delta^{-\hat{y}}(\mathbf{r})\right] / 4 .
$$

However, the strength of the induced $\Delta^{s}$ order is vanishingly small compared to the primary d-wave order, and hence it will be ignored in further discussions.

Antiferromagnetic Order: In the absence of an external magnetic field, the antiferromagnetic order is stabilized at half filling $(\langle n\rangle=1)$ and away from half filling, AFM order is quickly suppressed.
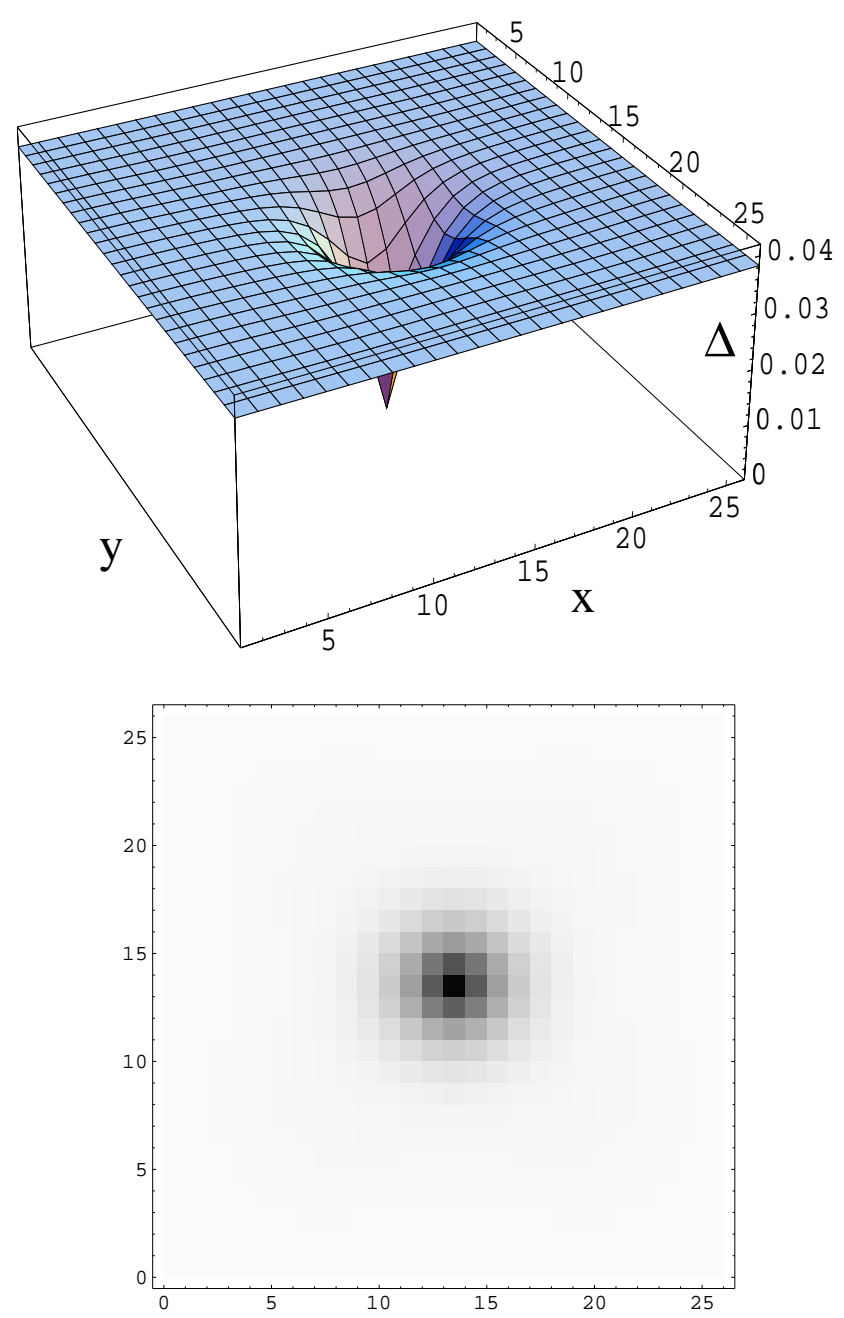

FIG. 1. Upper panel: Singlet d-wave pairing amplitude $\Delta_{i}$ on a magnetic unit cell of size $N=26 \times 26$, with $J=1.15 t$ and $\langle n\rangle=0.875 . \Delta_{i}$ is uniform (and equal to the BCS value) away from the vortex core, and falls to zero at the vortex center with the length scale $\xi$.

Lower panel: The grey-scale plot shows the length scale $\xi$. The dark (light) regions indicate smaller (larger) values for $\Delta_{i}$. Note that only half of the unit cell, containing one flux quantum, is shown here (and in all other figures). The full unit cell configuration can be obtained by periodically repeating the results along $\hat{y}$-direction.

For our choice of parameters, the minimum energy configuration is one in which AFM order is identically zero for the uniform system at zero external magnetic field $(H=0)$. However, at low temperatures, in the presence of a magnetic field, AFM order develops self-consistently in the vicinity of the vortex cores where superconductivity is destroyed. The structure of the AFM order is very different from that predicted earlier [17]. We have confirmed that the structure of our order parameter, which changes sign within the unit cell as is discussed below, minimizes the ground state energy, $E_{g}$, the expectation value of the Hamiltonian of Eq. (11), evaluated in the 
mean field ground state.
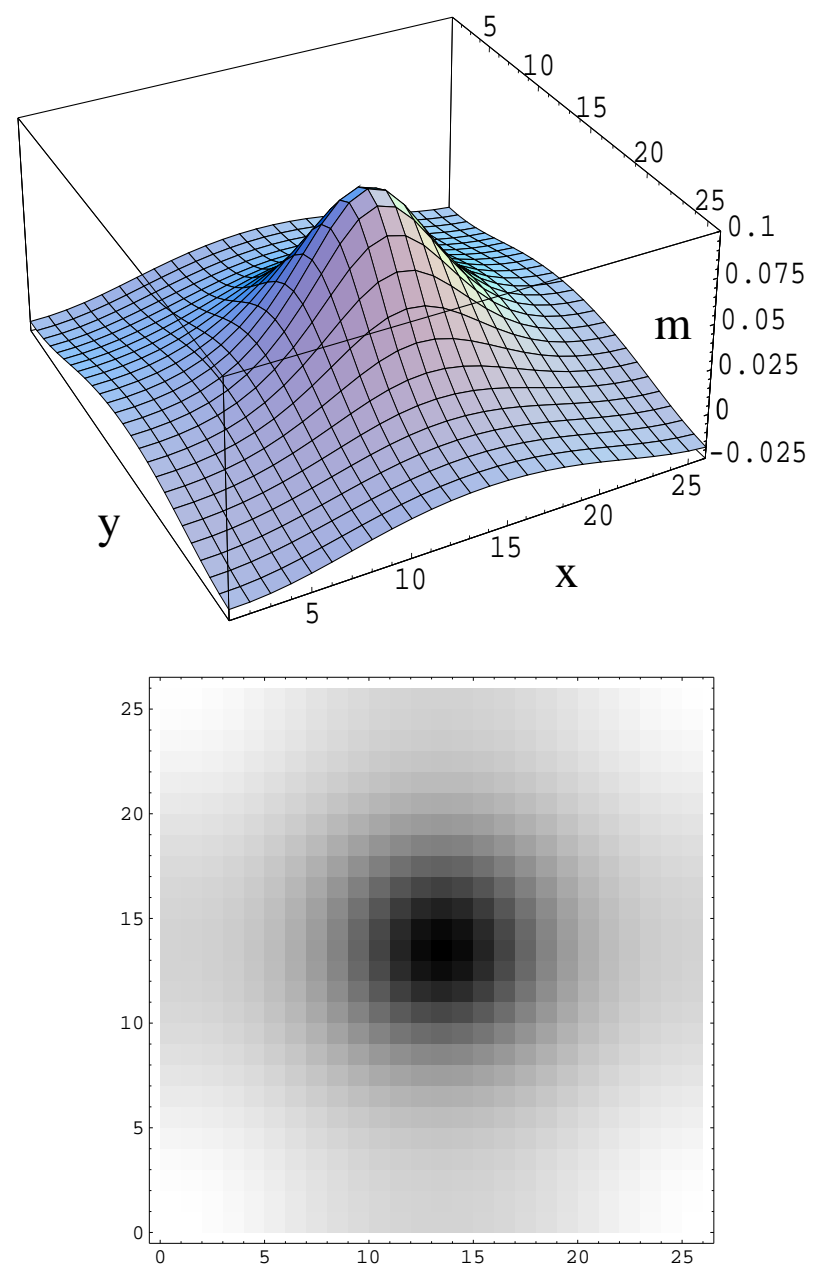

FIG. 2. Upper panel: Staggered magnetization $m_{i}$ on a magnetic unit cell of size $N=26 \times 26$ system, with $J=1.15 t$ and $\langle n\rangle=0.875 . m_{i}$ is strongest in the vortex cores, and decays very slowly away from it.

Lower panel: The grey-scale plot shows the much larger length scale for the decay of $m_{i}$ compared to $\xi$ (See also Fig. 1). The dark (light) regions indicate larger (smaller) values for $m_{i}$.

In this regard, we emphasize the role of including the Hartree and Fock shift terms. In a variational calculation, such as the BdG method, it is possible to achieve self-consistency for various different order parameter configurations corresponding to different $E_{g}$ 's. The correct configuration is the one that minimizes $E_{g}$. Ignoring the Hartree and Fock shifts will give a higher value for $E_{g}$.

The order parameter configuration in Ref. [17], when periodically repeated for an array of unit cells along $\hat{x}$ and $\hat{y}$ directions, produces an AFM order that changes sign on alternate unit cells along the $\hat{y}$-direction, and has the same sign along $\hat{x}$-direction. Such a "stripe-like" AFM order spontaneously breaks the symmetry of the $\hat{x}$ and $\hat{y}$ directions. Working with a unit cell containing 4 vortices, we have verified that such a "stripe-like"
AFM order does not converge to a self-consistent solution within our Hartree-Fock, BdG formalism. On the other hand, a "checkerboard" pattern of AFM order, in which the sign of AFM order alternates in adjacent unit cells along both the $\hat{x}$ and $\hat{y}$ directions, leads to a selfconsistent solution that has slightly higher $E_{g}$ than for the periodic configuration reported here.

Comparison of Fig. (2) to the result for the d-wave order parameter shown in Fig. (1i) above, clearly shows that the length scale of decay of the AFM order away from vortex core is much larger than the vortex core size $\xi$, as one would expect in the regime where uniform dSC is stable in the absence of an applied magnetic field.
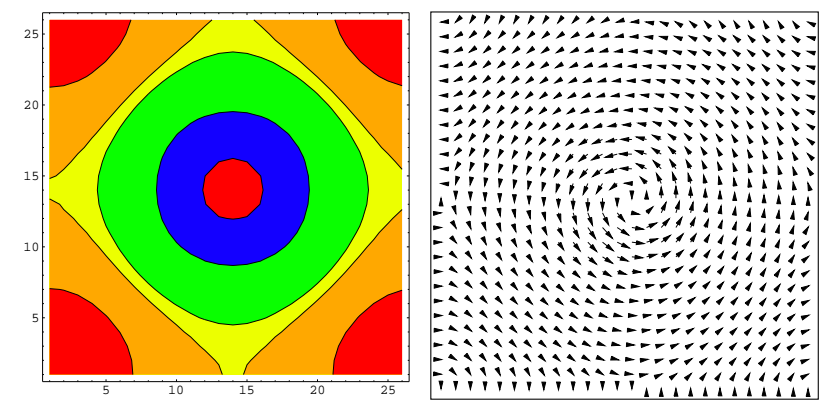

FIG. 3. Contour plot of staggered magnetization $m_{i}$ (left panel) on a magnetic unit cell of size $N=26 \times 26$ system, which shows that $m_{i}$ changes sign creating a domain wall along the line on which $\nabla \times j_{s} \approx 0$. The contours correspond to $m=0.075,0.03,0.004,0,-0.01$ from the center to radially outwards. Here, $j_{s}$ is the supercurrent associated with dSC order, which is plotted in the right panel. Notice that along the line where $m_{i} \approx 0, j_{s}$ arrows do not bend, showing that $\nabla \times j_{s} \approx 0$ along those lines.

We also notice in Fig. (3) the surprising result that the staggered magnetization changes sign within the vortex unit cell. Specifically it changes sign along the line where the supercurrent $j_{s}$ associated with the dSC order parameter has a zero curl, i.e. the line along which $j_{s}$ changes the direction of its winding. This result is true for a wide range of parameters studied (i.e. $J,\langle n\rangle$ and system size or external field strength); as well as for the study with the extended Hubbard model described in Sec. [I). This puzzling effect can be thought of as the result of an effective interaction of the following form: 28] $(\nabla \times \mathbf{j})_{I}(\nabla \times \mathbf{j})_{J} m_{I} m_{J}$, where $I$ and $J$ label adjacent plaquettes and $m_{I}$ is a course-grained AFM order associated with the plaquette. Alternatively, this implies that the gradient term for the AFM order in the G-L free energy takes the form $|(\nabla-a) m|^{2}$, where $a$ is proportional to the supercurrent. Note, that this interaction implies no preferred sign for the AFM order, but only that it changes sign along the line where the supercurrent vorticity changes sign. We have confirmed that this is also the case for our numerical BdG solution, i.e. there is no preferred direction for the AFM order. 
Charge Density Order: In the absence of AFM order the charge density is relatively structureless; $\left\langle n_{i}\right\rangle$ is uniform everywhere except very near a vortex. Around the vortex it has a very weak dip which changes to a weak peak at the vortex center. We believe that such weak density fluctuations would not survive long range Coulomb screening effects and hence will not have any important effects in a more realistic model. However, when the AFM order is allowed to develop self consistently, the charge density reorganizes itself significantly to accommodate the AFM order, as shown in Fig. (11).
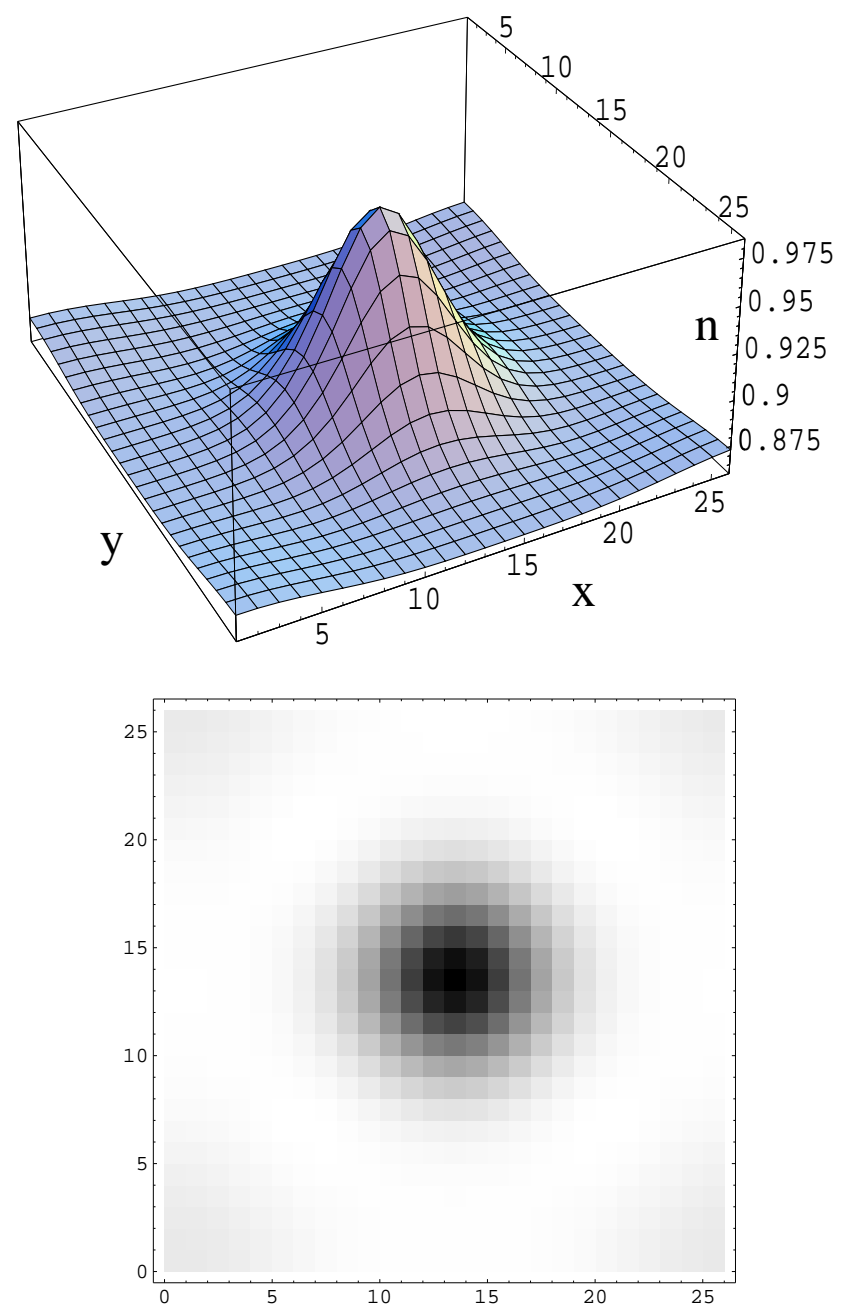

FIG. 4. Upper panel: Charge density $\left\langle n_{i}\right\rangle$ on a magnetic unit cell of size $N=26 \times 26$ system, with $J=1.15 t$ and $\langle n\rangle=0.875$. $\left\langle n_{i}\right\rangle$ is more or less uniform away from the vortex core, but achieves a value very close to half-filling $\left(\left\langle n_{i}\right\rangle=1\right)$ at the vortex center. This local half-filling at the vortex core favors the AFM order in the core region where dSC order is depleted due to external magnetic field.

Lower panel: The grey-scale plot shows that the distance from the vortex center where $\left\langle n_{i}\right\rangle$ differs significantly from its uniform value is slightly larger than $\xi$. (See also Fig. 1.) The dark (light) regions indicate larger (smaller) values for $\left\langle n_{i}\right\rangle$.
The local electron density remains more or less uniform away from the vortex center, but near the vortex core the magnitude of $\left\langle n_{i}\right\rangle$ increases continuously resulting in a density close to half filling at the vortex center. For the $t-J$ model at $T=0$, AFM order becomes the dominant order at half filling in the absence of the magnetic field. The charge density thus organizes itself to favor the AFM order strongly at the vortex core by approaching unity. Again, our result is different from that in Ref. [17], where $\left\langle n_{i}\right\rangle$ differs from the uniform bulk value only within one lattice spacing of the vortex center. Full self-consistency, including the Hartree and Fock terms, is required to reliably study charge inhomogeneities. However, one does need to keep in mind that the long-range Coloumb interaction in a three-dimensional material can further alter the charge distribution in ways which are beyond the scope of the present study.

Other self-consistent local variables such as the Hartree and Fock shift terms (not shown here) also become weakly inhomogeneous around the vortices.

$\pi$-triplet Order Parameter: In the presence of coexisting dSC and AFM orders, it had been argued [32.34 that an additional order, called $\pi$-triplet order, will develop self-consistently in the absence of any external magnetic field. It was shown using mean-field gap-equations that even in the absence of any interaction that generates $\pi$-triplet pairing directly, this order will be induced by coexisting DSC and AFM order. We define local $\pi$-triplet order as:

$$
\Pi_{i}^{\delta}=\frac{J}{4}\left\{\left\langle c_{i+\delta \downarrow} c_{i \uparrow}\right\rangle-\left\langle c_{i \downarrow} c_{i+\delta \uparrow}\right\rangle\right\} e^{i \mathbf{Q} \cdot \mathbf{r}_{i}} .
$$

This defines a triplet pairing amplitude in the $S_{z}=0$ channel. In the uniform system, Fourier transformation of $\Pi_{i}^{\delta}$ results in $\left\langle c_{-\mathbf{k} \downarrow} c_{\mathbf{k}+\mathbf{Q} \uparrow}\right\rangle$, so that the center of mass momentum for the pair is $\mathbf{Q}=(\pi, \pi)$, and hence the name. (The prefactor $J / 4$ is arbitrary in the absence of any microscopic interaction that generates this order, we use this prefactor to compare the strength of this order relative to $\Delta_{i}^{\delta}$.) One physical way of understanding the induction of the $\pi$-triplet order is the folowing. The dSC quasiparticles carry momenta $(\mathbf{k} \uparrow,-\mathbf{k} \downarrow)$, whereas the AFM quasiparticles carry momenta $(\mathbf{k}+\mathbf{Q} \uparrow, \mathbf{k} \uparrow)$. As a result, in the presence of both dSC and AFM order, there is a possibility of pairing between quasiparticles with momenta $(\mathbf{k}+\mathbf{Q} \uparrow,-\mathbf{k} \downarrow)$ which leads to the $\pi$ triplet pairing.

Our $t-J$ Hamiltonian does not contain any interaction involving $\Pi_{i}^{\delta}$ at the mean field level (Eq. Ф), but the BdG self-consistency induces $\Pi_{i}^{\delta}$ around the vortex, as shown in Fig. (5). On the other hand, the extended Hubbard model supports $\pi$-triplet pairing, and such an interaction should be present in the microscopic mean-field Hamiltonian equivalent to Eq. (位). We have verified that the qualitative results from the extended Hubbard model are similar to the results presented here. 

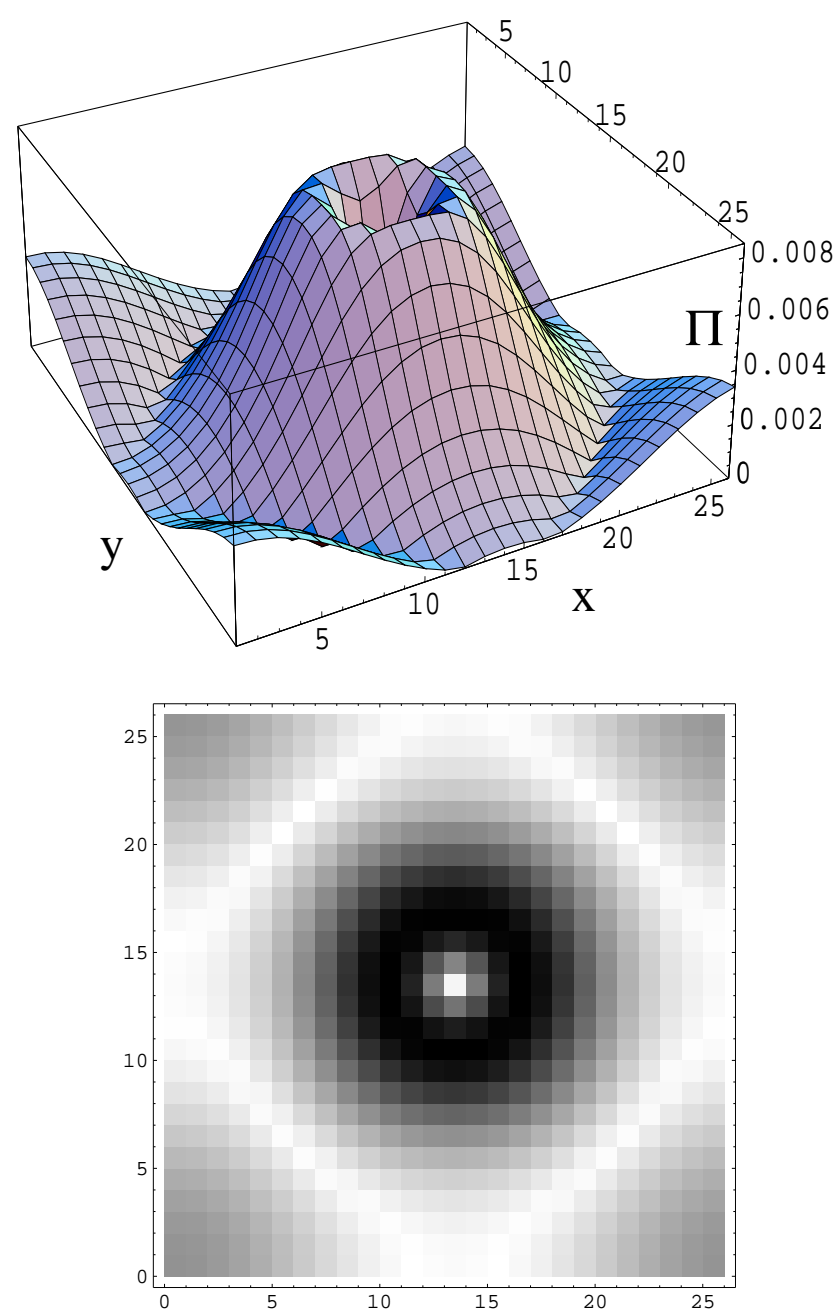

FIG. 5. Upper panel: $\pi$-triplet pairing amplitude $\Pi_{i}$ induced on a magnetic unit cell of size $N=26 \times 26$ system, with $J=1.15 t$ and $\langle n\rangle=0.875 . \Pi_{i}$ is zero at the vortex core, rises around the vortex, and falls gradually at larger distances.

Lower panel: The grey-scale plot for $\pi$-triplet showing the length scale of decay which is similar to the AFM order (in Fig. 2). The dark (light) regions indicate larger (smaller) values for $\Pi_{i}$. $\Pi_{i}$ also goes to zero on the lines of zero supercurrent.

This $\Pi_{i}^{\delta}$ order parameter was first introduced by Zhang [26] in a consistent picture for AFM and $\mathrm{dSC}$ order for cuprates using $\mathrm{SO}(5)$ theory. It was shown using general symmetry arguments that a theory which allows $\Delta_{i}^{\delta}, \Pi_{i}^{\delta *}$ and $m_{i}$ orders will satisfy the relation:

$$
\tilde{\Delta}_{i}^{\delta} \tilde{\Pi_{i}^{\delta} *}=-\tilde{m}_{i}\left(1-\left\langle n_{i}\right\rangle\right)
$$

where the variables with 'tilde' are the same as in Eq. (5) and in Eq. (14), but without the prefactors involving the coupling strength. This relation can be understood using a simpler picture of a so-called "spin-flop" model of an easy-axis AFM in a parallel magnetic field. Zhang 26] discussed the analogy between his $\mathrm{SO}(5)$ model and the spin-flop model, where for small fields the equilibrium state is one with an AFM order parameter, $N_{z}$, along the easy axis. As the field is increased above the spinflop transition, the Neel vector flops into the plane with a value $N_{\perp}$ and there is also a uniform magnetization, $M_{z}$, along the field. The case in which dSC and AFM order coexist corresponds to one in which the Neel vector $\vec{N}=\left(N_{\perp}, N_{z}\right)$ is tipped out of the plane and the uniform magnetization $\vec{M}=\left(M_{\perp}, M_{z}\right)$ is tipped away from the easy axis. $M_{\perp}$ is analogous to the $\Pi$ triplet order parameter. Since $\vec{N}$ is the difference of the two sublattice magnetizations, while $\vec{M}$ is their sum, $\vec{N} \cdot \vec{M}=0$. Using the correspondence between the two models, $m \leftrightarrow$ $N_{z}, \Delta \leftrightarrow N_{\perp},\left(1-\left\langle n_{i}\right\rangle\right) \equiv n_{H} \leftrightarrow M_{z}, \Pi \leftrightarrow M_{\perp}$, this implies the relation of Eq. (15).

As seen from Fig. (4), $\left\langle n_{\text {vortex }}\right\rangle \approx 0.975$ at the vortex center and hence does not achieve a value of true half-filling. However, calculations with increasing system sizes indicate that with larger system sizes the value approaches closer to $\left\langle n_{i}\right\rangle=1$. Defining the local hole density as $\left(\left\langle n_{\text {vortex }}\right\rangle-\left\langle n_{i}\right\rangle\right)$, instead of $\left(1-\left\langle n_{i}\right\rangle\right)$, we found that Eq. (15) is satisfied within 5\% accuracy. Although it is not obvious that our model has the same symmetry as the spin-flop model, we nevertheless find that this non-trivial relationship (Eq.15) holds rather well site by site!

\section{B. Local Density of States (LDOS) at the vortex core}

Having understood the self-consistent spatial structures of the relevant order parameters in an antiferromagnetic d-wave vortex, we turn our attention to the local density of states around the core. Study of the LDOS provides information about the low energy quasiparticle structure which is responsible for most of the novel phenomena associated with the cuprates. Also the LDOS is proportional to the tunneling conductance that is measured directly in STM experiments [0, 19] and hence can provide a direct test for the validity of theoretical predictions. As discussed above, a pure d-wave BCS mean field calculation [4] produces a large, unobserved ZBCP in the LDOS at the core of a d-wave vortex lattice. The ZBCP can be understood as the effect of Andreev reflection of d-wave quasiparticles within the vortex core. Andreev reflection is caused by the rotation of the internal state of an excitation from particle-type to hole-type (or vice versa) [33]. For a d-wave order parameter, such reflections scramble the phase information of the order parameter, and, as a result, the coherence peaks of the dSC collapse onto a ZBCP in the LDOS at the vortex core. Though the destruction of coherence peaks will occur for a wide range of angle for a reflecting surface, the effect is most drastic for the angle of $\pi / 4$. The obvious question is: Does the additional AFM order suppress the unphysical ZBCP at the vortex core? To address this issue, we present the LDOS at the vortex core as obtained 
from our calculations.

The single particle local density of states at $T=0$ is calculated from the expression:

$$
\begin{aligned}
N(i, \omega) & =\frac{1}{\mathcal{N}} \sum_{n, \mathbf{k}, \alpha}\left\{\left|u_{i, \alpha}^{n}(\mathbf{k})\right|^{2} \delta\left(\omega-E_{\alpha}^{n}(\mathbf{k})\right)\right. \\
& \left.+\left|v_{i, \alpha}^{n}(\mathbf{k})\right|^{2} \delta\left(\omega+E_{\alpha}^{n}(\mathbf{k})\right)\right\}
\end{aligned}
$$

where the individual delta functions are broadened with a width comparable to the average energy level spacing. The results are shown in Fig.(河).

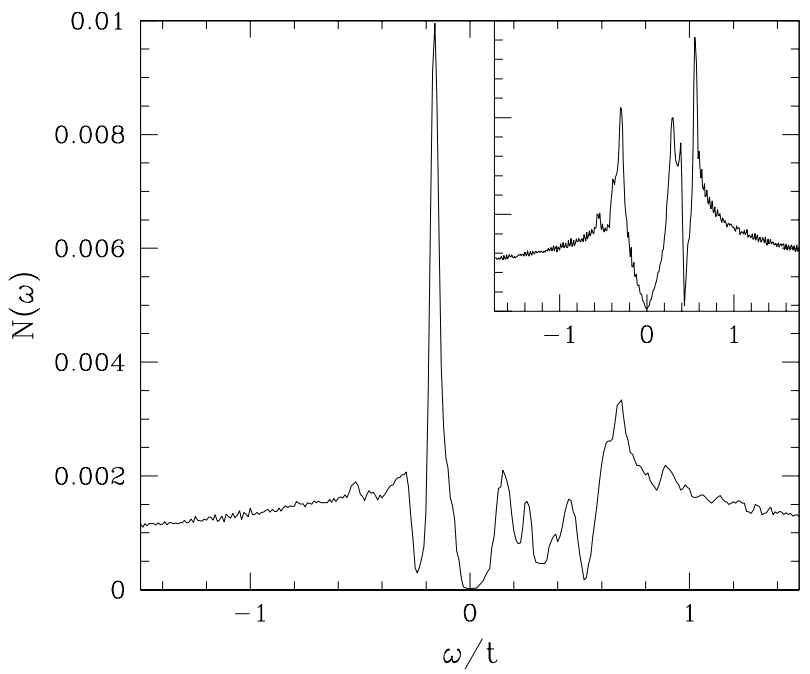

FIG. 6. Normalized density of states $N(\omega)$ at the vortex core for $J=1.15 t$ and $\langle n\rangle=0.875$. The competition of $\mathrm{dSC}$ and AFM order splits the zero bias peak which would be present in the absence of AFM order. The signature of a spin gap appears at large $\omega \sim 0.5$. The inset shows the DOS for a uniform system in the absence of vortices $(\hat{y}$-axis levels are not given for clarity). The parameters for the inset are $\Delta_{\max }=0.36, \mu=0.35$ and $m=0.1$, consistent with BdG results.

There are two important features in Fig.(6) that require further explanations. (i) The zero bias peak splits in two, and the coherence peaks are suppressed. (ii) An extra feature appears at large positive $\omega$ which looks like a second gap. Also, $N(\omega)$ appears to be quite asymmetric.

The asymmetry and the second gap scale (at $\omega \sim 0.5 t$ ) in the LDOS can be understood by analyzing the problem of a uniform d-wave SC coexisting with AFM order in the absence of vortex. This is described by the Hamiltonian $H=\sum_{\mathbf{k}}^{\prime} \Psi_{\mathbf{k}}^{*} M_{\mathbf{k}} \Psi_{\mathbf{k}}$ where,

$$
M_{\mathbf{k}}=\left(\begin{array}{cccc}
\xi_{\mathbf{k}}-\mu & \Delta_{\mathbf{k}} & -m & \Pi_{\mathbf{k}} \\
\Delta_{\mathbf{k}} & -\xi_{\mathbf{k}}+\mu & -\Pi_{\mathbf{k}} & -m \\
-m & -\Pi_{\mathbf{k}} & -\xi_{\mathbf{k}}-\mu & -\Delta_{\mathbf{k}} \\
\Pi_{\mathbf{k}} & -m & -\Delta_{\mathbf{k}} & \xi_{\mathbf{k}}+\mu
\end{array}\right)
$$

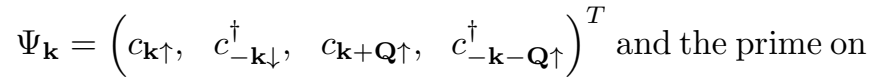
the $\mathbf{k}$-sum indicates a sum over only half of the Brillouin zone, which has been reduced by AFM order. Here $\xi_{\mathbf{k}}=$ $-2 t\left(\cos k_{x}+\cos k_{y}\right), \Delta_{\mathbf{k}}=\Delta_{0}\left(\cos k_{x}-\cos k_{y}\right)$ and $\Pi_{\mathbf{k}}=$ $\Pi_{0}\left(\cos k_{x}-\cos k_{y}\right)$. The result of the spin-density wave (SDW) problem with $\Delta_{0}=0, \Pi_{0}=0$ and $\mu=0$ is well known; under these conditions diagonalization of Eq. (17) results in a $N(\omega)$ that has a spin gap of size $m$ at the Fermi energy. Inclusion of particle hole asymmetry $(\mu \neq$ $0)$ shifts the overall density of states by an energy, $\mu$. Superconductivity can now be introduced with a nonzero $\Delta_{0}$, which will open a superconducting gap at $\omega=$ 0 with d-wave symmetry. The resulting DOS for this uniform system, which is plotted as an inset of Fig. (6), shows two gap scales, one at $\omega=0$ and the other at $\omega=$ $|\mu|+m$. With $m=0$, a non-zero $\Pi_{0}$ in Eq. (17) splits the dSC coherence peaks at $\Delta_{\max } \pm \Pi_{0}$; the splitting becomes asymmetric when $\mu \neq 0$. However, the $\pi$-triplet order does not change $N(\omega)$ qualitatively from that in the inset of Fig. (6) (at least for the choice of our parameters). (See also Fig. (10) of Ref. [34].) The presence of the vortex modifies the LDOS structure near $\omega=0$ (described in the next paragraph), but the SDW gap at larger $\omega$ survives the vortex excitations and shows up in Fig. (6). The above argumnet for the origin of the 'spin-gap scale' was verified by tuning model parameters that change $\mu$ and $m$. It was found that the 'spin-gap' changes accordingly in our BdG solutions.

Next we turn our attention to the splitting of the zero bias peak in $N(\omega)$. We have already argued (See Sec. II) that the ZBCP in the LDOS in Ref. [A] is due to the Andreev reflection of d-wave quasiparticles from the vortex core which acts like a boundary. The effects of an additional subdominant ordering on the ZBCP at the surface have been investigated recently 36 39. The conclusion is that, if the subdominant order breaks time-reversal symmetry, then the ZBCP will split in two. For our case, the subdominant order is AFM order, which breaks time-reversal symmetry, so that the presence of the AFM order within vortex core should split the ZBCP, as found in Fig. (6). Within this formalism, the energy difference between the split peaks would be proportional to the value of $m_{i}$ at the vortex core, which is consistent with our findings.

Having understood the features of the LDOS at the vortex core as found in our calculations, we must emphasize that, a mean-field calculation on a model with static antiferromagnetism like ours, does not reproduce all the experimental findings. Although the competition between dSC and AFM order produces a gap near the Fermi energy in the DOS - a result consistent with experimental findings - the spin-gap at larger $\omega$ has not been observed in experiments. Also, the strong splitpeak around $\omega=0$, which is due to static AFM does not resemble the weak subgap hump found in tunneling conductance data. The strong split peaks, however, die off rapidly away from the vortex center, as seen from the top panel of Fig. (7), which shows the evolution of the LDOS along the diagonal direction from the vortex. We also find that the coherence peaks build up near $\omega \sim \Delta_{\max }$ 
away from the core, as expected. Similar behaviour is found along the $\hat{x}$ and $\hat{y}$ directions as well.

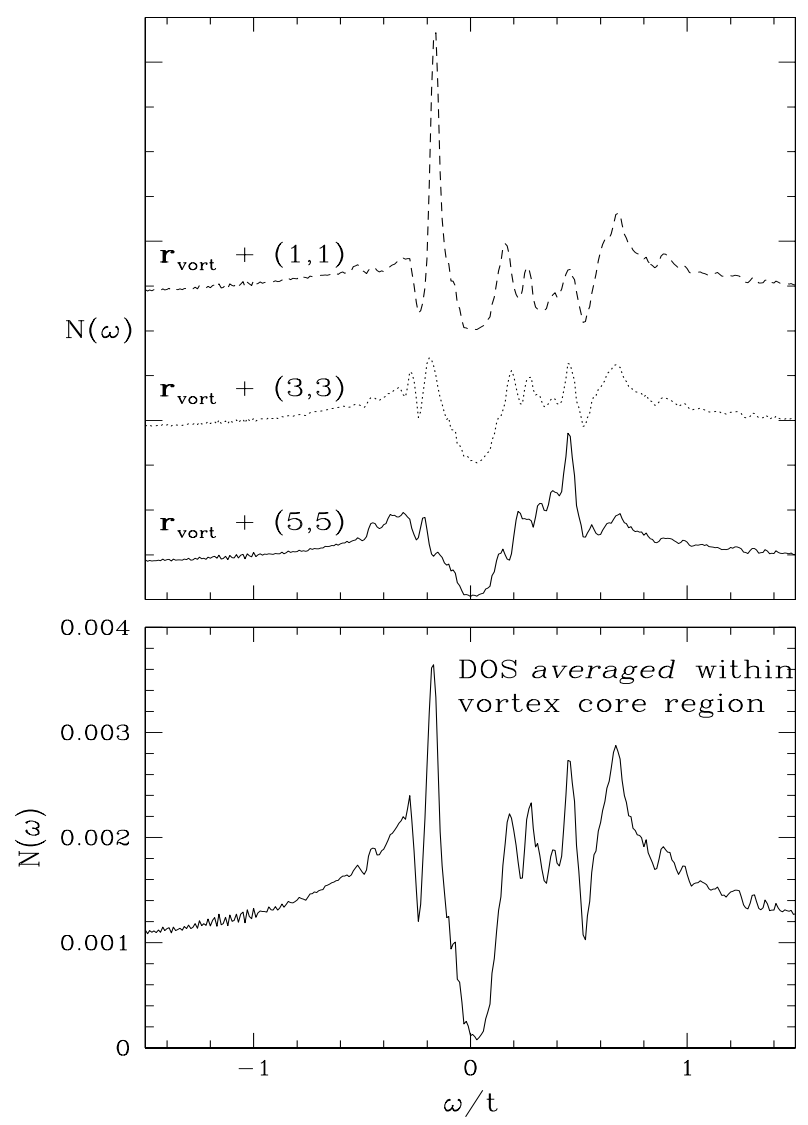

FIG. 7. Upper panel: Local Density of states away from the vortex core along the diagonal direction. The strong split-peaks fall rapidly in magnitude away from the vortex center.

Lower panel: Normalized local density of states averaged over all sites within a radius $\xi$ from the vortex center.

Surprisingly the low energy peaks persist to larger distances, even outside the vortex core. This is interesting from the experimental point of view, because such low energy quasiparticle humps have been observed beyond the vortex core [19]. This led us to calculate the local density of states averaged over all sites within a radius $\xi$ from the vortex core, which is presented in the lower panel of Fig. (7). Allowing for some uncertainty in locating the vortex center in STM measurements, we believe the qualitative agreement of our results in Fig. (7) with experimental findings is significant.

\section{CONCLUSIONS}

In conclusion, we have studied the spatial structures of different order parameters and their effect on the local density of states at the vortex core within a model that allows for the competition between d-wave superconduc- tivity and static antiferromagnetism. Our main results are: (1) Antiferromagnetic order develops near the vortex center, where the d-wave order parameter is suppressed, and exhibits interesting structure within the magnetic unit cell. (2) The length scale for decay of AFM order is larger than the superconducting coherence length. (3) Coexistence of dSC + AFM order induces $\pi$-triplet order in the vortex core region. (4) Competition between dSC and AFM splits the zero bias peak in the tunneling conductance. Experimental data strongly suggest that, at the vortex core, the low energy states near gap nodes are moved to higher energies. We believe that such physics can not be fully captured within mean-field theory. Dynamic AFM fluctuations and strong correlation effects should be included to make a more detailed comparison to the experimental data.

\section{Appendix}

\section{Self-consistent Parameters at the Boundary}

The boundary terms corresponding to Eq. 10 will be modified by making the following replacement on the left hand side of Eq. (10),

$$
\begin{gathered}
u_{i \pm \hat{x}, \alpha}^{n}(\mathbf{k}) \rightarrow u_{i \pm \hat{x} \mp N_{x} \hat{x}, \alpha}^{n}(\mathbf{k}) e^{\mp i k_{x} N_{x}} e^{\mp i a i_{y} N_{x}} \\
u_{i \pm \hat{y}, \alpha}^{n}(\mathbf{k}) \rightarrow u_{i \pm \hat{y} \mp N_{y} \hat{y}, \alpha}^{n}(\mathbf{k}) e^{\mp i k_{y} N_{y}} e^{\mp i a i_{x}} .
\end{gathered}
$$

and the definition of $\Delta_{\mathbf{q} n}^{\delta}(i)$ will be modified in the boundary as following:

$$
\begin{aligned}
\Delta_{\mathbf{q} n}^{ \pm \hat{x}}(i) & =\frac{J e^{ \pm i a i_{y} N_{x}}}{4 \mathcal{N}}\left[\left\{e^{\mp i q_{x} N_{x}} u_{i \pm \hat{x} \mp N_{x} \hat{x}, \downarrow}^{n}(\mathbf{q}) v_{i, \uparrow}^{n *}(\mathbf{q})\right.\right. \\
& \left.+e^{ \pm i k_{x} N_{x}} u_{i, \downarrow}^{n}(\mathbf{q}) v_{i \pm \hat{x} \mp N_{x} \hat{x}, \uparrow}^{n *}(\mathbf{q})\right\}\left(1-f_{\mathbf{q} n \downarrow}\right) \\
& +\left\{e^{\mp i k_{x} N_{x}} u_{i, \uparrow}^{n}(\mathbf{q}) v_{i \pm \hat{x} \mp N_{x} \hat{x}, \downarrow}^{n *}(\mathbf{q})\right. \\
& \left.\left.+e^{ \pm i k_{x} N_{x}} u_{j, \uparrow}^{n}(\mathbf{q}) v_{i, \downarrow}^{n *}(\mathbf{q})\right\} f_{\mathbf{k} n \uparrow}\right] \\
\Delta_{\mathbf{q} n}^{ \pm \hat{y}}(i) & =\frac{J}{4 \mathcal{N}}\left[\left\{e^{\mp i q_{y} N_{y}} u_{i \pm \hat{x} \mp N_{y} \hat{y}, \downarrow}^{n}(\mathbf{q}) v_{i, \uparrow}^{n *}(\mathbf{q})\right.\right. \\
& \left.+e^{ \pm i q_{y} N_{y}} u_{i, \downarrow}^{n}(\mathbf{q}) v_{i \pm \hat{x} \mp N_{y} \hat{y}, \uparrow}^{n *}(\mathbf{q})\right\}\left(1-f_{\mathbf{q} n \downarrow}\right) \\
& +\left\{e^{\mp i q_{y} N_{y}} u_{i, \uparrow}^{n}(\mathbf{q}) v_{i \pm \hat{x} \mp N_{y} \hat{y}, \downarrow}^{n *}(\mathbf{q})\right. \\
& \left.\left.+e^{ \pm i q_{y} N_{y}} u_{j, \uparrow}^{n}(\mathbf{q}) v_{i, \downarrow}^{n *}(\mathbf{k})\right\} f_{\mathbf{q} n \uparrow}\right]
\end{aligned}
$$

Self-consistency conditions for Hartree and Fock shifts (equivalent for Eq. (12)) are not given here; they are easily derivable in our formalism.

Acknowledgements: We would like to thank Eugene Kim, Henrik Bruus and, in particular, Shoucheng Zhang for many helpful discussions and suggestions. This work was supported by NSERC, the Canadian Institute for Advanced Research and SHARCNet. 
[1] C. Caroli, P. G. de Gennes and J. Matricon, Phys. Lett. 9, 307 (1964).

[2] F. Gygi and M. Schluter, Phys. Rev. B 43, 7609 (1991).

[3] H. Hess, R. B. Robinson and J. V. Waszczak, Phys. Rev. Lett., 64, 2711 (1990).

[4] Y. Wang and A. H. MacDonald, Phys. Rev. B 52, R3876 (1995).

[5] M. Ichioka, N. Hayashi, N. Enomoto and K. Machida, Phys. Rev. B 53, 15316 (1996).

[6] I. Maggio-Aprile, Ch. Renner, A. Erb, E. Walker and $\varnothing$. Fischer, Phys. Rev. Lett., 75, 2754 (1995).

[7] S.H. Pan et al., Phys. Rev. Lett., 85, 1536 (2000).

[8] M. Franz and Z. Tesanović, Phys. Rev. B 63, 64516 (2001).

[9] Q.-H. Wang, H. H. Han and D.-H. Lee, Phys. Rev. Lett., 87, 167004 (2001).

[10] J. H. Han and D.-H. Lee, Phys. Rev. Lett., 85, 1100 (2000).

[11] M. Franz and Z. Tesanović, Phys. Rev. Lett., 80, 4763 (1998).

[12] C. Wu, T. Xiang and Z.-B. Su, Phys. Rev. B 62, 14427 (2000).

[13] C. Berthod and B. Giovannini, Phys. Rev. Lett., 87, 277002 (2001).

[14] D. P. Arovas, A. J. Berlinsky, C. Kallin and S.-C. Zhang, Phys. Rev. Lett., 79, 2871 (1997).

[15] B. M. Andersen, H. Bruus and P. Hedegaard, Phys. Rev. B61, 6298 (2000).

[16] E. Demler, S. Sachdev and Y. Zhang, Phys. Rev. Lett., 87, 67202 (2001); S. Sachdev and S.-C. Zhang, Science, 295, 452 (2002); Y. Zhang, E. Demler and S. Sachdev, arXiv:cond-mat/0112343; A. Polkovnikov, M. Vojta and S. Sachdev, arXiv:cond-mat/0203176.

[17] J.-X. Zhu and C. S. Ting, Phys. Rev. Lett., 87, 147002 (2001).

[18] B. Lake et al., Science, 291, 1759 (2001).

[19] J. E. Hoffman et al., Science, 295, 466 (2002).

[20] B. Khaykovich et al., arXiv:cond-mat/0112505.

[21] V. F. Mitrovic et al., arXiv:cond-mat/0202368; V. F. Mitrovic et. al., Nature, 413, 501, (2001).

[22] R. I. Miller et al., Phys. Rev. Lett., 88, 137002 (2002).

[23] J.-X. Zhu, Ivar Martin and A. R. Bishop, arXiv:condmat/0201519.

[24] M. Franz, D. E. Sheehy and Z. Teanović, Phys. Rev. Lett., 88, 257005, (2002).

[25] Han-Dong Chen, Jiang-Ping Hu, Sylvain Capponi, Enrico Arrigoni and Shou-Cheng Zhang, arXiv:condmat/0203332

[26] S.-C. Zhang, Science, 275, 1089 (1997).

[27] B. Kyung and A. M. S. Tremblay, arXiv:condmat/0204500.

[28] S.-C. Zhang, constraint reference.

[29] Y. Chen and C. S. Ting, arXiv:cond-mat/0112369.

[30] S. Alama, A. J. Berlinsky, L. Bronsard and T. Giorgi, Phys. Rev. B 60, 6901 (1999).

[31] J-P. Hu and S-C. Zhang, arXiv:cond-mat/0108273.

[32] B. Kyung, Phys. Rev. B 62, 9083 (2000).

[33] A. F. Andreev, Zh. Eksp. Teor. Fiz. 46, 182 (1964); G.E. Blonder, M. Tinkham and T. M. Klapwijk, B 25, 4515 (1982).
[34] M. Murakami and H. Fukuyama, J. Phys. Soc. Jpn. 67, 2784 (1998).

[35] M. Matsumoto and H. Shiba, J. Phys. Soc. Jpn. 64, 1703 (1995).

[36] M. Matsumoto and H. Shiba, J. Phys. Soc. Jpn. 64, 3384 (1995); 64, 4867 (1995).

[37] M. Sigrist, D. B. Bailey and R. B. Laughlin, Phys. Rev. Lett., 74, 3249 (1995).

[38] D. Rainer, H. Burkhardt, M. Fogelstrom and J. A. Sauls, arXiv:cond-mat/9712234.

[39] L. J. Buchholtz, M. Palumbo, D. Rainer and J. A. Sauls, J. Low. Temp. Phys. 101, 1079 (1995); 101, 1099 (1995). 